\title{
ESTRATEGIAS PEDAGÓGICAS PARA UNA MEJOR ENSEÑANZA DE ESTUDIOS SUPERIORES EN DERECHO A ESTUDIANTES “MAYORES”.
}

\author{
Diana HERnÁNDEZ De La GuARdia \\ Prof. e Investigadora Auxiliar \\ dhguardia@minjus.gob.cu,refrigeración@sodeca.co.cu \\ Centro de Investigaciones Jurídicas
}

\begin{abstract}
Resumen:
Existe preocupación científica por mejorar la docencia a los estudiantes de Derecho, pero mayoritariamente son entendidos como tales los del sector muy juvenil. Del total de la bibliografía consultada, apenas se pudo encontrar trabajos interesados por esa otra porción del alumnado de más edad, por ello se considera que es tiempo de, aprovechando que existen posibilidades serias de mejoramiento, trabajar con ese fin. El artículo se estructura sobre la base de una investigación efectuada en Cuba y aplicada a estudiantes "mayores" de estudios superiores, es decir, de la Licenciatura en Derecho antes de comenzar el primer curso y después de culminado. Se identificaron algunos de los principales problemas que les preocuparon al iniciar los estudios superiores, se exponen cuáles son consideradas como posibles soluciones y se intenta brindar pautas a tener en cuenta en el perfeccionamiento de este sector de la enseñanza.
\end{abstract}

Palabras clave: pedagogía, conocimiento, universidad, docencia, Cuba.

\begin{abstract}
:
Scientists' concern for improving the pedagogy to the students of Law already exists but it is focused mainly on common young students. In the consulted bibliography there are only few papers regarding the students of more age, those who we call "older". This article shows a research regarding older Cuban students before beginning the first course of Degree in Law and after having finished the whole course. There were identified some of the troubles that worried them when beginning the studies and their possible solutions. A guideline for improve the teaching of older students is proposed.
\end{abstract}

Words key: pedagogy, knowledge, university, older students, Cuba.

Sumario: 1. Introducción. 2. Desazones iniciales. 2.1. Carencias de los alumnos mayores. 2.2. Pobre información científica sobre la enseñanza de Derecho para mayores. 2.3. Necesidad de preparar al profesorado con los nuevos conceptos. 3. Acciones para enfrentar las dificultades. 3.1. Medidas generales. 3.1.1. Tener en cuenta las características específicas del alumnado. 3.1.2. Romper esquemas con la impartición de una docencia no tradicional. 3.1.3. Preeminencia de contribuir a desarrollar habilidades para aprehender el conocimiento desde el inicio de los estudios. 3.1.4. Enriquecimiento de la pedagogía especializada con experiencias foráneas. 3.1.5. Contribuir a la formación futura de manera integral. 3.2. En cuanto a las características de las clases. 3.2.1. Escoger temas iniciales seductores. 3.2.2. Utilización de maneras gráficas como recomiendan los estudiantes y también la literatura. 3.2.3. Limitar el empleo de conferencias magistrales. 3.2.4. Énfasis en vincular la teoría REJIE Nueva época: Revista Jurídica de Investigación e Innovación Educativa Núm.18, Junio 2018, pp. 57-79 [En línea] http://www.revistas.uma.es/index.php/rejie 
con situaciones de actualidad. 3.2.5. Crear un clima interactivo. 3.2.6. Tomar en cuenta los avances en neuroeducación. 3.2.7. Monitorear cómo el estudiantado percibe la enseñanza. 3.2.8. Imprescindible presencia en la docencia de la interdisciplinariedad, la multidisciplinariedad y la transdisciplinariedad. 3.2.9. Reverenciar la enseñanza que dé prioridad a los valores, a los principios éticos. 3.2.10. Aprovechar el interés por estudiar del estudiantado mayor. 3.2.11. Convertir el proceso docente educativo en un sistema armónico donde la enseñanza tenga correspondencia con la forma de evaluación. 4. Otras propuestas para lograr los resultados esperados. 4.1. Utilización de métodos de enseñanza en correspondencia con los objetivos docentes propuestos y las necesidades del alumnado mayor. 4.2. Utilización de los TICs. 4.3. Tratamiento diferenciado de la enseñanza a mayores. 5. Conclusiones. Bibliografía.

\section{Introducción.}

Los temas pedagógicos en ciencias jurídicas están mayormente enfocados hacia un sector muy juvenil. Del total de la bibliografía consultada, apenas se pudo encontrar trabajos que se interesen por el alumnado de mayor edad, entonces ¿cómo contribuir al aprendizaje de los que sobrepasan esa media, a los que llamaremos "mayores”? ¿Puede manejarse ese grupo de igual manera que el primero o debe tenerse en cuenta la disparidad? ¿Las diferencias implicarían el uso de sistemas de enseñanza únicos para este sector -lo cual llevaría a un rompimiento con los mecanismos pedagógicos actuales- o la utilización de manera creativa y diferenciada de los ya existentes?

Como en muchos otros países ${ }^{1}$, en Cuba los estudios universitarios comprenden principalmente: Licenciatura, Maestría y Doctorado. Para acceder a cada uno de ellos se realiza un proceso de evaluación y selección. Se valoran aptitudes y destrezas de los postulantes vinculado a los niveles a que aspire, aunque siempre se trata de una enseñanza totalmente gratuita.

Con el interés de contribuir al mejoramiento de la enseñanza superior de mayores, se ha elaborado este artículo basado en una investigación realizada en Cuba y aplicada a estos estudiantes de la Licenciatura en Derecho (por tanto, no se incluye el resto de las modalidades), antes de comenzar el primer curso recibido y después de culminado, por lo cual ellos identificaron algunos de los principales problemas que les preocuparon al iniciar los estudios superiores, se exponen cuáles son consideradas como posibles soluciones y se intenta brindar algunas pautas a tener en cuenta en el perfeccionamiento de la enseñanza de este sector.

Por tanto, el trabajo consta de una primera parte donde se exponen las falencias y las dificultades que afrontan tanto los estudiantes -para llevar a feliz término sus estudios superiores-, como las que pueden afectar al profesorado, que intenta atemperarse a los nuevos tiempos y brindar una docencia de calidad.

\footnotetext{
${ }^{1}$ Elías Méndez, C. (2010). "Análisis de los textos y de la normativa de referencia en relación con el proceso de Bolonia”, en Cotino Hueso, Lorenzo y PRESNO LinerA, Miguel (coords.), Innovación educativa en Derecho constitucional. Reflexiones, métodos y experiencias de los docentes, PUV (Servicio de Publicaciones de la Universidad de Valencia), Valencia, pp. 20-21, http: //www.uv.es/derechos/innovacionconstitucional.pdf.
} 
En una segunda parte se explicitan las acciones que pueden contribuir a resolver los problemas planteados y se culmina con un panorama conciso de algunas de las pautas a tener en cuenta para el mejoramiento de la enseñanza superior de mayores, sobre la base de considerar necesario brindarle un tratamiento diferenciado, si bien se ha de remarcar que en nuestra opinión, la solución no ha de transitar por un quebrantamiento o transformación radical de los mecanismos pedagógicos existentes, sean estos los más tradicionales o los evidentemente innovadores, sino que las respuestas cada docente habrá de proyectarlas a partir de una caracterización de su colectivo estudiantil y la utilización de manera imaginativa de los mecanismos que le permitan enriquecer y mejorar el aprendizaje de estos.

Para nada el sentido de optimar la enseñanza de mayores puede conducir a crear compartimentos estancos desprovistos de conexión con la rica experiencia pedagógica acumulada a lo largo de los tantos años de formación de juristas. Todo lo contrario, se ha de beber del saber y la experiencia existente, valioso tesoro al cual acudir con creatividad y sentido holístico. El trabajo solo pretende dar una imagen de algunos de los variados mecanismos tradicionales y de innovación pedagógica a fin de que los docentes, en función de las características de su alumnado, puedan aprehenderlos con sensibilidad y aplicarlos de forma particularizada cuando se trate de la enseñanza superior a mayores.

\section{Desazones iniciales.}

\subsection{Carencias de los alumnos mayores.}

Desde la cátedra de un alumnado mayor se puede admitir que si los jóvenes tienen dificultades, los de más edad las pueden tener también pero incrementadas. Los mayores muchas veces están confundidos sobre si realmente deben o no asumir el reto de realizar estudios superiores -ya que como la educación es gratuita, no pocas veces han llegado a las aulas compelidos por los centros laborales donde se desempeñan, los cuales han apreciado posibilidades de desarrollo de esos sujetos, pero limitaciones en su formación-, sabiendo que deben incrementar sus esfuerzos durante varios años de fuerte preparación académica.

Si los estudios previos no contribuyeron a crear una formación adecuada a los universitarios jóvenes, en función de enfrentar el reto de culminar una licenciatura y por ello cuando pisan las aulas universitarias perciben el cambio, en los mayores se da el caso que, al estar durante años desvinculados totalmente de los estudios, se muestran más afectados, pues a las carencias anteriores se suman las suyas propias.

Perdieron (si existió alguna vez) el hábito de estudiar, como también la capacidad de aprendizaje de sus años mozos, el método para prepararse adecuadamente para una asignatura o las destrezas para realizar un buen examen, en fin, no solo se trata del incremento en la complejidad de la materia a estudiar (Licenciatura), sino también de la manera de manejarla adecuadamente.

Sin embargo, este último elemento no fue identificado por los entrevistados. De un total de 47 alumnos mayores sujetos a la investigación, refirieron como las limitantes que les 
entorpecían aquellas vinculadas a la falta de tiempo para estudiar: el hecho de encontrarse laborando en puestos relevantes que les robaban muchísimo espacio, por lo cual no podían dedicar todo el que quisieran para sus carreras.

Reconocieron también que estudiar les obligaba a manejar demasiada información y ello les resultaba muy engorroso, pues requería una dedicación y un tiempo del cual no disponían, e incluían otras situaciones propias de la vida cotidiana, como la presencia de familiares a su responsabilidad y abrigo. Igualmente consideraron contribuía a empeorar la situación, la exigencia en desempeños laborales nuevos e importantes. Hubo un grupo que reflejó algo muy autóctono: las dificultades existentes en el país con la transportación de pasajeros en la ciudad, pues también les restaba posibilidades de estudio.

Los estudiantes valoraron que esos problemas podían ser solucionados si eran capaces de partir de ciertas pautas éticas, imprescindibles y grandemente vinculadas entre sí, como voluntad, interés, esfuerzo, perseverancia, dedicación y sacrificio.

En la investigación realizada se pusieron de manifiesto las dificultades que presentaban con el procesamiento de la información que se les brindaba, como por ejemplo, con la comprensión de lecturas, la determinación de las palabras claves, el resumen o la interpretación.

Expresaron que preferían la organización de esa información escrita a partir de la agrupación (por medio de llaves, plecas, etc.), y de diagramas, cuadros sinópticos, algoritmos, etc., a la presentación de textos interminables, tal vez porque actualmente predomina el suministro de información de esta manera y también, porque a falta de tiempo y de hábito de lectura, ello simplifica la actividad de aprendizaje ${ }^{2}$.

\subsection{Pobre información científica sobre la enseñanza de Derecho para mayores.}

Con el fin de ahondar en el tema, el primer paso fue, sin dudas, el de la pesquisa para conocer el estado del arte de la materia. ¡Cuánta no fue la sorpresa al constatar que podía considerarse casi nulo!

Las grandes dificultades que atraviesa el profesorado para llegar a los estudiantes jóvenes son patentizadas en los artículos analizados, donde se evidencia que aquellos están cada vez menos motivados, más disgregados con sus aditamentos electrónicos y menos preparados académicamente para la enseñanza superior ${ }^{3}$. También se abordan otros temas pedagógicos, pero es muy pobre la información para los estudiantes que nos preocupan. Muchas veces se habla genéricamente de "estudiantes", entre los cuales pueden ser incluidos, por supuesto, los mayores, pero se han encontrado apenas estudios

\footnotetext{
${ }^{2}$ IÑigo CORrozA, M. y SÁnChEZ- Ostiz GutiéRReZ, P. (2014). "Sobre el uso del método inductivo en la enseñanza del Derecho Penal", REJIE: Revista Jurídica de Investigación e Innovación Educativa, núm.9, p. 12.

3 PALAO TABOADA, C. (2002). "La enseñanza del derecho en la Universidad: presente y futuro", AFDUAM, núm. 6, pp.127-139.
} 
que traten específicamente sobre la enseñanza de ese sector ${ }^{4}$, lo cual, da una medida de la importancia de abordar la temática.

\subsection{Necesidad de preparar al profesorado con los nuevos conceptos.}

El profesor tiene ante sí supremos desafíos: debe dominar la materia a impartir, pero también debe hacerla atractiva a sus alumnos mayores. Como sobre sus hombros recae la motivación y el aprendizaje de estos, algunos países tienen implementados cursos para desarrollar las capacidades docentes ${ }^{5}$.

Y es que el sistema de enseñanza constituye un único proceso que debe funcionar como un todo. Para lograrlo se deben preparar a los docentes en los nuevos contenidos pedagógicos, insertarse con los alumnos en la moderna dinámica informática ${ }^{6}$, sin obviar la importancia de mejorar la organización y la planificación de la docencia ${ }^{7}$, determinar los instrumentos docentes a utilizar en relación con los objetivos que se pretenden alcanzar, así como seleccionar de forma mesurada los contenidos a impartir, para no caer en la saturación de materias y el rechazo del alumnado ${ }^{8}$.

Por supuesto, todas esas tareas representan destinar una mayor cantidad de tiempo a la actividad pedagógica en general ${ }^{9}$, dentro de la cual se incluye, como muy relevante, el rol que el profesor ha de desempeñar en las tutorías ${ }^{10}$, todo lo cual ha generado algunos agobios que no pocas veces son coronados con mayores éxitos.

\footnotetext{
${ }^{4}$ Escobar RocA, G. (2010). "Seis años de experiencia de formación virtual del programa regional de apoyo a las defensorías del pueblo de Iberoamérica”, en Cotino Hueso, Lorenzo y Presno Linera, Miguel (coords.), Innovación educativa en Derecho constitucional. Reflexiones, métodos y experiencias de los docentes, PUV (Servicio de Publicaciones de la Universidad de Valencia), Valencia, pp. 180-184, http://www.uv.es/derechos/innovacionconstitucional.pdf.

${ }^{5}$ KANDEL, V. N. (2010). "Cuando la universidad se enseña a sí misma. Notas sobre carrera docente academia”, Revista Sobre Enseñanza del Derecho, Buenos Aires, Argentina, año VIII, núm. 16, pp. 231239.

${ }^{6}$ SÁNCHEZ DE DiEgo, M. (2010). "Una visión crítica a partir de la experiencia en la gestión modificación de planes de estudio en ciencias de la información”, en Cotino HUESO, Lorenzo y PRESNO LiNERA, Miguel (coords.), Innovación educativa en Derecho constitucional. Reflexiones, métodos y experiencias de los docentes, PUV (Servicio de Publicaciones de la Universidad de Valencia), Valencia, p. 57, http://www.uv.es/derechos/innovacionconstitucional.pdf.

7 Martínez Alarcón, M. L. (2010). "Algunas reflexiones sobre la revisión del modelo docente universitario", en Cotino HuESO, Lorenzo y PRESNo LinerA, Miguel (coords.), Innovación educativa en Derecho constitucional. Reflexiones, métodos y experiencias de los docentes, PUV (Servicio de Publicaciones de la Universidad de Valencia), Valencia, p. 49, http://www.uv.es/derechos/innovacionconstitucional.pdf.

${ }^{8}$ IÑIgo Corroza, M. E. y SÁnCheZ- Ostiz GutiérReZ, P. (2014). "Sobre el uso del método inductivo en la enseñanza del Derecho Penal”, REJIE: Revista Jurídica de Investigación e Innovación Educativa, núm.9, p. 13.

9 RochA NÚÑEZ, E. (2011). “Aplicación del método ABP y la clínica procesal en la enseñanza aprendizaje del Derecho Procesal Mercantil”, RIDE: Revista Iberoamericana para la Investigación y el Desarrollo Educativo, vol. 1, núm. 2, enero - junio, p. 163. Vila RAMOS, B. (2011). "Una propuesta práctica de innovación docente: La enseñanza-aprendizaje-evaluación del Derecho Constitucional", Indret: Revista para el Análisis del Derecho, núm. 1, p. 28.

${ }^{10}$ Bueno Gallardo, E. (2013). "Formulación de una propuesta didáctica para la enseñanza-aprendizaje de la asignatura Derecho Financiero y Tributario I", Docencia y Derecho, Revista para la Docencia Jurídica Universitaria, núm. 7, pp. 15-16.
} 


\section{Acciones para enfrentar las dificultades.}

\subsection{Medidas generales.}

Siguiendo la línea trazada en la conformación de este estudio, se tomará en cuenta lo más relevante del acervo pedagógico actual, para exponer distintas posibles soluciones a las dificultades y retos que es necesario afrontar por el profesorado de estudiantes mayores.

\subsubsection{Tener en cuenta las características específicas del alumnado.}

Un proceso tan complejo y diverso como el de la enseñanza tiene que enriquecerse y adecuarse en el día a día. Para trabajar con los estudiantes mayores es preciso conocer sus particularidades y principales dificultades. Lo mejor es tratar de lograr que la enseñanza se realice de la manera más individualizada posible ${ }^{11}$. La aplicación al principio del curso de un sencillo cuestionario ${ }^{12}$ que permita caracterizarles y conocer sus preocupaciones, puede resultar de gran utilidad para proyectar una estrategia docente, como se constata en la investigación mencionada.

En Cuba, a diferencia de lo que se ha encontrado en la literatura acerca de la disminución de la cantidad del estudiantado en las escuelas de educación superior ${ }^{13}$, no se aprecia tal situación a pesar de la baja natalidad y del envejecimiento de la población. Se vivencian aulas llenas, con ávidos estudiantes mayores, colmados de carencias intelectuales diversas sobre las que es preciso trabajar con ahínco.

\subsubsection{Romper esquemas con la impartición de una docencia no tradicional.}

La enseñanza tradicional considera que el estudiante no conoce de la temática impartida, de modo que el profesor con su sapiencia le acerca a ésta para que aquel, distante del conocedor, logre incorporarla.

Pero ahora se trata de transformar ese papel elevado, protagonista y distante del profesor versado que explica y evalúa (sobre todo memorísticamente), para dar paso a un agente activo, que participa con el estudiante en el proceso educativo, estimulando lo mejor del alumnado con una adecuada conducción y retroalimentación en toda esa dinámica de construcción del conocimiento.

Como explica González Rus, se debe pasar del "modelo de enseñanza" al "modelo del aprendizaje", entendido por este autor como aquel que prepara al estudiante por medio

\footnotetext{
${ }^{11}$ Vila Ramos, B. (2011). "Una propuesta práctica de innovación docente: La enseñanza-aprendizajeevaluación del Derecho Constitucional”, Indret: Revista para el Análisis del Derecho, núm. 1, p. 27.

${ }^{12}$ Rubio NúñEZ, R. (2010). "La enseñanza virtual de un Derecho global”, en Cotino HuEso, Lorenzo y PRESNO LinerA, Miguel (coords.), Innovación educativa en Derecho constitucional. Reflexiones, métodos y experiencias de los docentes, PUV (Servicio de Publicaciones de la Universidad de Valencia), Valencia, p. 42, http://www.uv.es/derechos/innovacionconstitucional.pdf.

${ }^{13}$ Rubio NúÑEZ, R. (2010). "La enseñanza virtual de un Derecho global”, en Cotino HuESO, Lorenzo y PRESNO LinerA, Miguel (coords.), Innovación educativa en Derecho constitucional. Reflexiones, métodos y experiencias de los docentes, PUV (Servicio de Publicaciones de la Universidad de Valencia), Valencia, p. 34, http://www.uv.es/derechos/innovacionconstitucional.pdf.
} 
de técnicas, métodos y procedimientos para conocer el Derecho por sí mismo, identificar y resolver los problemas que plantea su interpretación y aplicación, de modo que sea su propia iniciativa, experiencia y esfuerzo formativo, lo que sustituya a lo que en el modelo de enseñanza recibe pasivamente ${ }^{14}$.

Ello implica grandes evoluciones en la función docente. No pocos titulan al método encargado de tal transformación, como método cooperativo, considerado más que nada una estrategia de enseñanza que incluye actividades activas del alumnado, ejecutadas en grupos, los cuales realizan tareas conjuntas como problemas, solución de ejercicios, estudio de casos, etc., con la ventaja que contribuyen a colocar al alumno en una posición activa, estimulando sus capacidades y preparándolo para su desempeño futuro $^{15}$.

Se trata de un cambio de concepción que abarca otras variables, pues deben tenerse en cuenta distintos elementos, como se expondrá a continuación.

\subsubsection{Preeminencia de contribuir a desarrollar habilidades para aprehender el conocimiento desde el inicio de los estudios.}

Como se expresó anteriormente, la generalidad de los estudiantes mayores acuden a las aulas luego de un tiempo sin vinculación docente. Es por eso que se propone se inserte desde el comienzo un curso, un adiestramiento, etc., que contribuya a que los alumnos adquieran destrezas para incrementar su potencial cognitivo. La Organización de las Naciones Unidas para la Educación, la Ciencia y la Cultura (UNESCO) ${ }^{16}$ ha recomendado reforzar esta capacidad.

Por medio de esos cursos, los mayores pueden identificar la manera mejor de apropiarse de la información, o lo que es lo mismo, determinar el camino por medio del cual se llega a un resultado positivo y consciente en el proceso de aprendizaje, de modo que pueda ser utilizado en otras ocasiones con autonomía, de forma racional, lo que ha sido llamado como "metacognición”, es decir, "el conocimiento y regulación que poseemos de todos estos procesos básicos, superiores y complejos: qué son, cómo operan, cuándo

\footnotetext{
${ }^{14}$ GonzÁlez Rus, J. J. (2003). "Reflexiones sobre el futuro de la enseñanza del Derecho y sobre la enseñanza del Derecho en el futuro", Revista Electrónica de Ciencia Penal y Criminología, (en línea), núm. 05-r1, p. r1:1-r1:21, http://criminet.ugr.es/recpc/recpc05-r1.pdf

15 Peñas Moyano, M. J. et al. (2010). "Métodos cooperativos en la enseñanza del derecho: una aproximación al derecho mercantil en las aulas de facultades no jurídicas", Revista de educación y derecho. EDUCATION AND LAW REVIEW, núm. 2, abril - septiembre, pp. 1-21. IÑIGO CORROZA, M. E. y SÁncheZ- Ostiz GutiérRez, P. (2014). "Sobre el uso del método inductivo en la enseñanza del Derecho Penal”, REJIE: Revista Jurídica de Investigación e Innovación Educativa, núm. 9, p. 19.

${ }^{16}$ Weinstein, J. (1998). "La búsqueda del tesoro perdido. Educación y Juventud en América Latina", Boletín Proyecto Principal de Educación en América Latina y el Caribe, núm. 45, abril, pp. 71-87. AMADIO, Massimo, et al. (2013). "Ejes de formación y enfoques curriculares", Oficina Internacional de Educación de la UNESCO (Ginebra, Suiza), http://www.unesco.org/new/es/santiago/pressroom/newsletters/newsletter-laboratory-for-assessment-of-the-quality-of-education-llece/n12/trainingareas-and-curriculum-approaches/
} 
hay que utilizar uno u otro, qué factores coadyuvan o entorpecen su funcionamiento, etc." ${ }^{17}$.

De ese modo, con esas herramientas, de forma conjunta y con una visión amplia, el profesor y los alumnos colaboran, develan y construyen juntos los caminos del aprendizaje, al mostrarle al alumno el proceso que puede utilizar para estudiar mejor, con vistas al desarrollo de sus capacidades y valores. En Cuba se ha instituido una asignatura con el nombre de "Aprender a Aprender", para ayudar en esta cuestión a los estudiantes mayores.

\subsubsection{Enriquecimiento de la pedagogía especializada con experiencias foráneas.}

En nuestro mundo cada vez más globalizado, resulta imprescindible conocer y adecuar los conocimientos y prácticas pedagógicas que otros han instituido de manera exitosa, para sopesar su implementación en los procederes vernáculos. Específicamente y para el área iberoamericana, que tantos puntos de contacto tiene en materia jurídica, constituye más que una recomendación, una necesidad.

No hay límites, es más, es tanta la interacción a nivel global, que aunque el sistema jurídico vigente tenga diferencias marcadas con otros, como el del common law, por ejemplo, no implica que no tengamos mucho que aprender también de sus experiencias pedagógicas. De hecho, prácticas como los concursos Moot -en los que participan estudiantes de numerosos centros de educación superior- dan fe de ello. En esos eventos se trabaja con un "caso de ficción", el cual "es realizado por... la Universidad de Buenos Aires, ...(así como)... la Universidad del Rosario de Bogotá, Colombia y ... participan cada año cerca de 50 universidades de América, de Estados Unidos y de Europa, tal es el caso de la Universidad de Miami y Sciences Po en París"18.

\subsubsection{Contribuir a la formación futura de manera integral.}

El objetivo final de la enseñanza a los estudiantes mayores es lograr que lleguen a convertirse en juristas plenos. Con vistas a garantizar el desempeño futuro, a estos estudiantes de Derecho se les deben desarrollar destrezas de distinto tipo, teniendo en cuenta que la carrera puede demandar de ellos habilidades oratorias, en la escritura, en el pensamiento lógico, etc. La utilización en la práctica docente cotidiana de mecanismos de participación y diversos métodos de enseñanza, contribuirá a suscitar en ellos las reflexiones y favorecerá acertadas decisiones.

\footnotetext{
${ }^{17}$ Alama Flores, C. M. (2015). "Hacia una didáctica de la metacognición”, Horizonte de la Ciencia, Junín, Perú, vol. 5, núm. 8, pp.77-86, http://www.uncp.edu.pe/revistas/index.php/horizontedelaciencia/issue/view/9.

${ }^{18}$ Consolo, A. N. (2015). "El Arbitraje Comercial Internacional y los Moot Practice. Los métodos que se utilizan en la enseñanza de las Ciencias Jurídicas y Sociales", Derecho y ciencias sociales, La Plata, núm. 13, octubre, pp. 3-12.
} 


\subsection{En cuanto a las características de las clases.}

\subsubsection{Escoger temas iniciales seductores.}

Siguiendo los cánones de la enseñanza tradicional, las materias se imparten de un modo lógico y progresivo, de lo general a lo particular, de lo simple a lo complejo. Sin demeritar las ventajas pedagógicas de tal presentación, es fundamental atraer desde el comienzo al alumnado mayor con temas llamativos, aunque no se siga de modo ortodoxo el camino usual ${ }^{19}$. En un ambiente relajado y con ideas atractivas se puede lograr mucho. Hay un viejo refrán que reza: nunca hay una segunda ocasión para causar una buena primera impresión.

\subsubsection{Utilización de maneras gráficas como recomiendan los estudiantes y también la literatura.}

Para nadie es un secreto que las generaciones actuales, aunque tengan cierta edad, en su mayoría son muy "visuales", por lo cual reciben con agrado clases donde se presenta la información agrupada ${ }^{20}$ en llaves, plecas, diagramas, cuadros sinópticos, etc. (como se apuntó), así como insertadas con el uso de fragmentos de películas, de documentales ${ }^{21}$, juicios grabados, presentaciones de youtube $e^{22}$, etc., lo que equivaldría a "penetrar en la fortaleza audiovisual de los alumnos con sus mismas armas" 23 , siempre teniendo presente la participación activa del profesor al seleccionar los audiovisuales o partes de éstos, realizar la presentación ante el auditorio así como fomentar al final la discusión y el debate.

\footnotetext{
19 Torres Villarreal, M. L. (2013). "La enseñanza clínica del derecho: una forma de educación para el cambio social. La experiencia del grupo de acciones públicas de la Universidad del Rosario", Revista Facultad de Derecho y Ciencias Políticas, Medellín, vol. 43, núm. 119, enero-junio, pp. 725-726.

${ }^{20}$ Rubio NúÑEZ, R. (2010). "La enseñanza virtual de un Derecho global”, en Cotino Hueso, Lorenzo y PRESNO LINERA, Miguel (coords.), Innovación educativa en Derecho constitucional. Reflexiones, métodos y experiencias de los docentes, PUV (Servicio de Publicaciones de la Universidad de Valencia), Valencia, p. 42, http://www.uv.es/derechos/innovacionconstitucional.pdf.

${ }^{21}$ TAJADURA, J. (2010). "La utilización de medios audiovisuales en las clases prácticas de Derecho Constitucional", en Cotino Hueso, Lorenzo y PRESNO LinERA, Miguel (coords.), Innovación educativa en Derecho constitucional. Reflexiones, métodos y experiencias de los docentes, PUV (Servicio de Publicaciones de la Universidad de Valencia), Valencia, http: //www.uv.es/derechos/innovacionconstitucional.pdf, pp. 192-198. Oliveros AYA, C. (2010). "El cine político: un recurso didáctico en la enseñanza del derecho constitucional", Diálogos de saberes: investigaciones y ciencias sociales, núm. 33, pp. 245-260. VILA RAMOS, B. (2011). "Una propuesta práctica de innovación docente: La enseñanza-aprendizaje-evaluación del Derecho Constitucional", Indret: Revista para el Análisis del Derecho, España, núm. 1, p. 23. CoRREDOIRA, L. (2010). "El uso de la IP TV en la docencia de Derecho de la Información”, en COTINO HUESO, Lorenzo y PRESNO LINERA, Miguel (coords.), Innovación educativa en Derecho constitucional. Reflexiones, métodos y experiencias de los docentes, PUV (Servicio de Publicaciones de la Universidad de Valencia), Valencia, pp. 199 y ss., http: //www.uv.es/derechos/innovacionconstitucional.pdf.

${ }^{22}$ Vila RAmOS, B. (2011). "Una propuesta práctica de innovación docente: La enseñanza-aprendizajeevaluación del Derecho Constitucional”, Indret: Revista para el Análisis del Derecho, núm. 1, p. 24.

${ }^{23}$ Rubio NúñEZ, R. (2010). "La enseñanza virtual de un Derecho global”, en Cotino HuESO, Lorenzo y PRESNO LinerA, Miguel (coords.), Innovación educativa en Derecho constitucional. Reflexiones, métodos y experiencias de los docentes, PUV (Servicio de Publicaciones de la Universidad de Valencia), Valencia, p. 33, http://www.uv.es/derechos/innovacionconstitucional.pdf.
} 


\subsubsection{Limitar el empleo de conferencias magistrales.}

Si bien la utilización muy extendida durante un tiempo de este tipo de enseñanza ha ocasionado una crítica casi unánime ${ }^{24}$, debido a que establece un distanciamiento con el alumnado para convertirse el maestro en el impartidor por excelencia de la docencia con énfasis en la aplicación de recursos memorísticos-, es perceptible que aunque se limite tampoco se debe desechar su uso, pues constituye una manera eficaz de brindar conocimientos $^{25}$.

Para el caso de los estudiantes mayores, debe tenerse en cuenta que rechazan las disertaciones con terminologías rebuscadas. En el ambiente profesional se ensalza la buena oratoria, la cual lleva implícita el manejo de un amplio léxico, que de utilizarse con frecuencia se hace propio, pero al emplearlo en demasía afecta al alumnado, pues no recibe el mensaje esperado y queda con innumerables interrogantes sin resolver. Si el objetivo es que se capten los contenidos, no es acertado distanciarlo de ellos, al contrario, se les debe aproximar de la mejor manera y la más sencilla es utilizar con los mayores un vocabulario diáfano y llano.

Otra dificultad muy reconocida por los docentes, es el hecho que cada vez son más voluminosas las materias a impartir, lo cual limita a los profesores en la utilización de las nuevas metodologías docentes y atormenta a los alumnos con una carga casi insalvable para sus ojos $^{26}$. Deben encontrarse caminos más simples para brindar el contenido de las materias y hacerlo de una manera más factible ${ }^{27}$.

Debe remarcarse que el método tradicional de enseñanza utiliza mucho el ejercicio de la memoria y aunque se propugna la transformación de la impartición y el aprendizaje de conocimientos en Derecho con la disminución del uso excesivo de las exigencias memorísticas, se puede reconocer que tampoco se debe caer en su "desprestigio",28, dado que es imprescindible para fijar los conocimientos, para lo cual es necesario leer y

\footnotetext{
${ }^{24}$ IÑIgo CorroZA, M. E. y SÁNChEZ- Ostiz GutiéRreZ, P. (2014). "Sobre el uso del método inductivo en la enseñanza del Derecho Penal", REJIE: Revista Jurídica de Investigación e Innovación Educativa, núm. 9, p. 10. PÉrez CÁZARES, M. E. (2012). "Teoría de los conceptos en la enseñanza del Derecho en base a casos concretos", Academia. Revista sobre enseñanza del Derecho, Buenos Aires, año X, núm. 20, pp. 41-42.

${ }^{25}$ Martínez Alarcón, M. L. (2010). "Algunas reflexiones sobre la revisión del modelo docente universitario", en Cotino HuESO, Lorenzo y PRESNO LINERA, Miguel (coords.), Innovación educativa en Derecho constitucional. Reflexiones, métodos y experiencias de los docentes, PUV (Servicio de Publicaciones de la Universidad de Valencia), Valencia, pp. 49-50, http://www.uv.es/derechos/innovacionconstitucional.pdf. VARGAS VASSEROT, C. (2009). "El método del caso en la enseñanza del Derecho: experiencia piloto de un piloto novel", Refiedu: Revista de Formación e Innovación Educativa Universitaria, vol. 2, núm. 4, p. 205.

${ }^{26}$ Palao TABOAdA, C. (2002). "La enseñanza del derecho en la Universidad: presente y futuro", AFDUAM, núm. 6, pp. 127-139.

${ }^{27}$ IÑIgo CorrozA, M. E. y SÁnChEZ- Ostiz GutiérReZ, P. (2014). “Sobre el uso del método inductivo en la enseñanza del Derecho Penal”, REJIE: Revista Jurídica de Investigación e Innovación Educativa, núm. 9, p. 13.

${ }^{28}$ Rubio Núñez, R. (2010). "La enseñanza virtual de un Derecho global”, en Cotino HuEso, Lorenzo y PRESNO LinerA, Miguel (coords.), Innovación educativa en Derecho constitucional. Reflexiones, métodos y experiencias de los docentes, PUV (Servicio de Publicaciones de la Universidad de Valencia), Valencia, p. 35, http://www.uv.es/derechos/innovacionconstitucional.pdf.
} 
volver a leer, por tanto, este otro elemento (la estimulación a la lectura), es un factor muy positivo a su favor.

\subsection{4. Énfasis en vincular la teoría con situaciones de actualidad.}

No puede quedar a un lado la dogmática jurídica, pero a la vez, cuando es presentada desvinculada de la realidad se puede convertir en algo etéreo, cuando no vacío. Conscientes de esta limitante y a modo de ejemplo palpable de esta forma de pensar, en el Centro de Investigaciones Jurídicas fue elaborado un libro sobre Metodología de la Investigación Socio jurídica. El halago inequívocamente unánime recibido fue el haber presentado la teoría aderezada de interesantes ejemplos jurídicos.

E igual ocurre con las clases, los estudiantes mayores temen atiborrarse de información inservible, que ni comprenden bien ni vislumbran cómo puede ser utilizada, por eso agradecen los maestros que los motivan al vincular la teoría con recientes vivencias.

\subsubsection{Crear un clima interactivo.}

La añeja manera de presentar la información sin relacionarse con el estudiantado debe dar paso a clases dinámicas, en las que todos aprendan: alumnos y profesores, en ellas se debe estimular la creatividad, la solución de situaciones, la crítica y el análisis. Para ayudarse pueden ser utilizados artículos de la prensa, películas, documentales, etc. sobre los cuales se puede objetar y razonar.

\subsubsection{Tomar en cuenta los avances en neuroeducación.}

Este elemento se debe consignar por su importancia, pues estudios realizados han mostrado los cambios en los niveles de atención de los asistentes a clases. Si se conoce que se asimilan más conocimientos en los primeros quince a veinte minutos, se ha de priorizar ese momento para brindar a los alumnos aquello valorado como más importante, siempre que sea posible ${ }^{29}$.

Tal "descubrimiento" entra en contradicción con ciertas normas metodológicas diseñadas para la impartición de la docencia tradicional: el profesor en los minutos iniciales debe presentar el tema y los objetivos que se propone con su docencia, así como revisar colectivamente la tarea que haya quedado pendiente del encuentro anterior. Si no se cumple con ese orden "pedagógico", la clase es reprobada.

Pero siempre hay soluciones para congeniar ambos criterios: pudieran brindarse datos sobre el tema y los objetivos desde el final de la clase anterior, utilizar la forma escrita para revisar la tarea y luego dar los resultados, en fin, habrá de encontrarse variantes a asumir en bien de los educandos.

\subsubsection{Monitorear cómo el estudiantado percibe la enseñanza.}

No hay manera de conocer la apreciación del alumnado acerca de los cambios que se están introduciendo, si no se les aplica algún cuestionario anónimo por medio del cual

\footnotetext{
${ }^{29}$ CODINA Felip, M. J. (2015). Neuroeducación en virtudes cordiales: cómo reconciliar lo que decimos con lo que hacemos, Barcelona, Ediciones Octaedro, S.L., pp. 91 y ss.
} 
se pueda evaluar cómo se ha recibido la docencia y cómo se valoran los cambios ${ }^{30}$. La aplicación de la técnica del PNI (positivo, negativo e interesante) ha resultado muy provechosa, pues ha permitido conocer concretamente, aquello que debe ser superado.

En la investigación mencionada y como resultado de la utilización del PNI, se conoció que si bien los estudiantes mayores identificaron como positivos o interesantes los métodos de enseñanza utilizados, la forma de organizar e impartir las clases, el énfasis en la consolidación de valores así como la dinámica del trabajo en equipo, se sintieron afectados por las dificultades presentadas con los medios audiovisuales en las clases, el exceso de textos en las presentaciones de power point e interesaron se ampliasen la cantidad de horas lectivas.

\subsubsection{Imprescindible presencia en la docencia de la interdisciplinariedad, la multidisciplinariedad y la transdisciplinariedad.}

Ningún docente desea que de sus aulas salgan "licenciados tullidos" 31 : es necesario desarrollar competencias que faculten a los graduados a desempeñarse con acierto en su futura actividad laboral, a gozar de adecuada habilidad en la escritura, como en la expresión oral, tener lo que ha sido llamado acertadamente como "conciencia o juicio crítico",32, pues es muy valioso contar con juristas capaces de tener sus propias opiniones, de analizar de forma mesurada y profunda lo que acontece así como de formarse una opinión defendible con argumentaciones.

Para lograrlo no basta únicamente con el Derecho, se necesita sumar los saberes de otras ciencias con enfoques multi, inter y trans disciplinarios, imprescindibles en la enseñanza de los asuntos jurídicos ${ }^{33}$ y en esa línea de pensamiento, algunos proponen para contribuir a una aplicación más plena de las normas y mejorar la docencia, la participación conjunta con pedagogos, psicólogos, profesores de teatro, de expresión corporal $^{34}$, sociólogos ${ }^{35}$, etc.

\footnotetext{
${ }^{30}$ IÑIgo CorrozA, M. E. y SÁnChEZ- Ostiz GutiérReZ, P. (2014). "Sobre el uso del método inductivo en la enseñanza del Derecho Penal”, REJIE: Revista Jurídica de Investigación e Innovación Educativa, núm. 9 , p. 19.

${ }^{31}$ Nieto, A. (2005). “La enseñanza académica del Derecho”, Lex Nova, julio- septiembre, pp. 13, 27.

${ }^{32}$ Vila RAmos, B. (2011). "Una propuesta práctica de innovación docente: La enseñanza-aprendizajeevaluación del Derecho Constitucional”, Indret: Revista para el Análisis del Derecho, núm. 1, pp. 23-24, 30 .

${ }^{33}$ LARrauri TORroella, R. (2006). "La enseñanza del concepto de derecho desde la Filosofía del Derecho contemporánea", Academia. Revista sobre Enseñanza del Derecho, Buenos Aires, año IV, núm. 7, pp. 159 y ss. Serrano PÉrez, M. M. y MARTí SÁnChez, J. M. (2010). "La planificación y desarrollo compartido de competencias", en COTINo HUESO, Lorenzo y PRESNO LiNERA, Miguel (coords.), Innovación educativa en Derecho constitucional. Reflexiones, métodos y experiencias de los docentes, PUV (Servicio de Publicaciones de la Universidad de Valencia), Valencia, p. 66, http: //www.uv.es/derechos/innovacionconstitucional.pdf. LEMES BATISTA, A. y CÁCERES GIRAUD, A. (2006). "Enfoque de la interdisciplinaridad y transdisciplinaridad en la enseñanza de la Economía Política”, Revista Iberoamericana de Educación, OEI, núm. 40/6, 15 de diciembre.

${ }^{34}$ TORRES VillarReal, M. L. (2013). "La enseñanza clínica del derecho: una forma de educación para el cambio social. La experiencia del grupo de acciones públicas de la Universidad del Rosario", Revista Facultad de Derecho y Ciencias Políticas, Medellín, vol. 43, núm. 119, enero-junio, p. 714.

${ }^{35}$ PAdrón InNAmorato, M. y CÁCERes Nieto, E. (2009). "Sociología jurídica y constructivismo: hacia una metodología para la determinación de la pensión alimenticia en el estado de Tabasco", Revista Latinoamericana de Derecho Social, núm. $9, \quad$ pp. $122 \quad$ y http://www.juridicas.unam.mx/publica/librev/rev/revlads/cont/9/art/art5.pdf
} 


\subsubsection{Reverenciar la enseñanza que dé prioridad a los valores, a los principios éticos.}

El Derecho es un fenómeno social y a su vez obliga a tomar partido, a pronunciarse sobre un suceso que ha acaecido, lo cual conlleva inevitablemente la estrecha interacción con la sociedad. La educación basada en valores privilegia lo mejor de la profesión letrada y potencia esenciales proyecciones ${ }^{36}$.

\subsubsection{Aprovechar el interés por estudiar del estudiantado mayor.}

Es frecuente la queja del profesorado de jóvenes acerca de que se muestran apáticos, poco motivados y morosos ${ }^{37}$. Se ha de reconocer que se ha observado lo contrario con aquellos de más edad: se les percibe muy interesados en captar los conocimientos, se muestran participativos, sin timideces y con mucha garra. Esos elementos positivos son muy valiosos y contribuyen al avance de los objetivos propuestos.

\subsubsection{Convertir el proceso docente educativo en un sistema armónico donde la enseñanza tenga correspondencia con la forma de evaluación}

Parece una verdad de Perogrullo pero no está de más apuntar la importancia de hacer corresponder la docencia con el método de validación adecuado, con énfasis en utilizar en las evaluaciones situaciones de la práctica, pues si eso se logra, los estudiantes se sienten parte del mundo jurídico ${ }^{38}$.

Las evaluaciones no deben convertirse en una sanción para los estudiantes. Pueden plantearse variantes que pueden oscilar entre las más tradicionales, a aquellas en las que se incorpora la utilización de los instrumentos electrónicos, las que retan a los alumnos, la evaluación continuada, las coevaluaciones, las evaluaciones de doble objeto (donde se evalúan tanto los conocimientos como las competencias) ${ }^{39}$, etc., hasta la supresión de una evaluación final si anteriormente se vencen ciertos parámetros ${ }^{40}$.

\footnotetext{
${ }^{36}$ DAVison M, D. (2006). "La visión jeffersoniana de la educación jurídica", Academia. Revista sobre Enseñanza del Derecho, Buenos Aires, año IV, núm. 7, pp. 47-49. Cotino Hueso, L. (2010). "No casos, sino 'pasos' en la enseñanza de derechos fundamentales", en COTINO HUESO, Lorenzo y PRESNO LINERA, Miguel (coords.), Innovación educativa en Derecho constitucional. Reflexiones, métodos y experiencias de los docentes, PUV (Servicio de Publicaciones de la Universidad de Valencia), Valencia, p. 102. http://www.uv.es/derechos/innovacionconstitucional.pdf.

37 SAlAzAR Benítez, O. (2010). "Estrategias y herramientas para la enseñanza del derecho constitucional", Docencia y Derecho, núm. 1, p. 9.

${ }^{38}$ RoCHA NúÑEZ, E. (2011). “Aplicación del método ABP y la clínica procesal en la enseñanza aprendizaje del Derecho Procesal Mercantil”, RIDE: Revista Iberoamericana para la Investigación y el Desarrollo Educativo, vol. 1, núm. 2, enero - junio, pp. 159.

${ }^{39}$ BuEno GAllardo, E. (2013). "Formulación de una propuesta didáctica para la enseñanza-aprendizaje de la asignatura Derecho Financiero y Tributario I", Docencia y Derecho, Revista para la Docencia Jurídica Universitaria, núm. 7, pp. 22-23, 26-29.

${ }^{40}$ SÁnCHEZ Ruiz, M. (2013). "Cuánto y cómo enseñar derecho mercantil en el grado en relaciones laborales y recursos humanos”, Trabajo. Revista andaluza de relaciones laborales, Universidad de Huelva, Huelva, núm. 29, pp. 85-99.
} 


\section{Otras propuestas para lograr los resultados esperados}

\subsection{Utilización de métodos de enseñanza en correspondencia con los objetivos docentes propuestos y las necesidades del alumnado mayor}

Son válidos todos los métodos, desde los más usuales, como las exposiciones orales, los debates, los análisis legislativos, la redacción de dictámenes ${ }^{41}$, etc., como otros no tan comunes como por ejemplo:

4.1.1. El aprendizaje basado en problemas ${ }^{42}$, que de forma abreviada se conoce como $\mathrm{ABP}$, con el cual se pueden vincular hechos de la vida cotidiana con las bases teóricas.

4.1.2. Dar participación a los "moot practice" 43 , los cuales se han convertido en todo un movimiento competitivo a nivel internacional. Surgió en los Estados Unidos y utiliza casos ficticios para estimular la creatividad, el análisis, la redacción y muy especialmente, la oratoria.

4.1.3. La utilización del diálogo socrático ${ }^{44}$, con el cual el profesor intentará mediante inteligentes y continuas preguntas a los estudiantes (y por supuesto, sin facilitar él las respuestas), favorecer el debate a partir de los planteamientos realizados y lograr así que los estudiantes razonen y lleguen al conocimiento, estimulando su capacidad reflexiva.

4.1.4. El juego de roles $^{45}$. Se asignan determinadas tareas profesionales a los estudiantes, quienes previamente deben preparar sus alegatos y respuestas a los planteamientos de los otros participantes. El profesor actúa como mediador en todo el proceso.

\footnotetext{
${ }^{41}$ Bueno Gallardo, E. (2013). "Formulación de una propuesta didáctica para la enseñanza-aprendizaje de la asignatura Derecho Financiero y Tributario I", Docencia y Derecho, Revista para la Docencia Jurídica Universitaria, núm. 7, p. 10.

${ }^{42}$ Rocha NúÑEZ, E. (2011). "Aplicación del método ABP y la clínica procesal en la enseñanza aprendizaje del Derecho Procesal Mercantil”, RIDE: Revista Iberoamericana para la Investigación y el Desarrollo Educativo, vol. 1, núm. 2, enero - junio, p. 164. VARGAS VASSEROT, C. (2009). "El método del caso en la enseñanza del Derecho: experiencia piloto de un piloto novel", Refiedu: Revista de Formación e Innovación Educativa Universitaria, vol. 2, núm. 4, p. 199.

${ }^{43}$ Consolo, AnAlía N. (2015). "El Arbitraje Comercial Internacional y los Moot Practice. Los métodos que se utilizan en la enseñanza de las Ciencias Jurídicas y Sociales", Derecho y ciencias sociales (Ejemplar dedicado a: Derecho y Ciencias Sociales), La Plata, núm. 13, octubre, pp. 3-12.

${ }^{44}$ Pires PinTo SiCA, L. P. et al. (2012). "Reflexiones sobre enseñanza del Derecho y evaluación en el contexto brasileño", Academia. Revista sobre enseñanza del Derecho, Buenos Aires, año X, núm. 20, pp. 64-65. Consolo, AnAlía N. (2015). "El Arbitraje Comercial Internacional y los Moot Practice. Los métodos que se utilizan en la enseñanza de las Ciencias Jurídicas y Sociales, Derecho y ciencias sociales", Editorial Arbitraje Comercial (Ejemplar dedicado a: Derecho y Ciencias Sociales), La Plata, núm. 13, octubre, p. 10. VARGAS VASSEROT, C. (2009). "El método del caso en la enseñanza del Derecho: experiencia piloto de un piloto novel", Refiedu: Revista de Formación e Innovación Educativa Universitaria, vol. 2, núm. 4, p. 198.

${ }^{45}$ Rocha NúÑEZ, E. (2011). "Aplicación del método ABP y la clínica procesal en la enseñanza aprendizaje del Derecho Procesal Mercantil”, RIDE: Revista Iberoamericana para la Investigación y el Desarrollo Educativo, vol. 1, núm. 2, enero - junio, p. 156. PIRES PINTO SICA, L. P. et al., (2012). "Reflexiones sobre enseñanza del Derecho y evaluación en el contexto brasileño", Academia: Revista sobre enseñanza del Derecho, Buenos Aires, año X, núm. 20, pp. 70-71. Vila RAmos, B. (2011). "Una propuesta práctica de innovación docente: La enseñanza-aprendizaje-evaluación del Derecho Constitucional", Indret: Revista para el Análisis del Derecho, núm. 1, p. 25.
} 
4.1.5. La solución de problemas interesantes ${ }^{46}$, incluida la utilización del método inductivo $^{47}$, con lo cual se estimula la enseñanza a partir de problemas y situaciones fácticas de las que se extraen los elementos generales, donde el profesor puede comenzar presentando un problema y brindar el camino para solucionarlo como ejemplo a seguir, o simplemente esbozando la situación que quiere se analice, como también puede aportar alternativas de solución para los casos muy complejos, fomentando la participación.

4.1.6. La clínica jurídica ${ }^{48}$. La denominación de esta técnica proviene del ambiente anglosajón, donde es usual que los estudiantes de Medicina acudan a los hospitales y clínicas para realizar prácticas médicas. Extrapolada al Derecho tal concepción, ha recibido igual denominación para los aprendizajes que ocurren en ambientes jurídicos como Tribunales, Colegios de Abogados, etc., pudiendo llegar a crearse locales o sedes (denominadas "clínicas jurídicas") a las cuales pueden concurrir personas de bajos recursos a recibir el asesoramiento de estudiantes, bajo la atenta dirección de los profesores ${ }^{49}$.

4.1.7. El estudio de $\operatorname{casos}^{50}$. Este antiguo y a veces minimizado método -proviene de la época romana-, busca acercar al jurista a la práctica, al presentar situaciones que han ocurrido en la actividad profesional. En la etapa moderna se plantea que fue el decano de la Universidad de Harvard, Christopher Columbus Langdell, quien en 1879 publicó un libro de casos y estimuló este método, hoy extendido.

4.1.8. El uso del método Jigsaw", (o "rompecabezas"), que fortalece el trabajo en equipo pero a la vez recurre al trabajo individual: en él cada participante debe

\footnotetext{
${ }^{46}$ Pires Pinto SiCA, L. P. et al., (2012). "Reflexiones sobre enseñanza del Derecho y evaluación en el contexto brasileño", Academia: Revista sobre enseñanza del Derecho, Buenos Aires, año X, núm. 20, pp. 67-68.

${ }^{47}$ IÑIgo CorrozA, M. E. y SÁnChEZ- OSTIZ GutiéRreZ, P. (2014). "Sobre el uso del método inductivo en la enseñanza del Derecho Penal", REJIE: Revista Jurídica de Investigación e Innovación Educativa, núm.9,pp. 9-22.

${ }^{48}$ Molina Saldarriaga, C. A. (2008). "Fundamentos teóricos y metodológicos del método clínico de enseñanza del derecho", Revista Facultad de Derecho y Ciencias Políticas, vol. 38, núm. 108, pp. 187214.Otros consideran que se acerca más al juego de roles: Torres VillarreAL, M. L. (2013). "La enseñanza clínica del derecho: una forma de educación para el cambio social. La experiencia del grupo de acciones públicas de la Universidad del Rosario", Revista Facultad de Derecho y Ciencias Políticas, Medellín, vol. 43, núm. 119, enero-junio. p. 711.

${ }^{49}$ TORRES Villarreal, M. L. (2013). "La enseñanza clínica del derecho: una forma de educación para el cambio social. La experiencia del grupo de acciones públicas de la Universidad del Rosario", Revista Facultad de Derecho y Ciencias Políticas, Medellín, vol. 43, núm. 119, enero-junio, p. 715.

${ }^{50}$ Zumaquero Gil, L. (2016). "Cómo enseñar derecho civil en la titulación de grado en Turismo a través del estudio de casos", REJIE: Revista Jurídica de Investigación e Innovación Educativa, núm. 13, pp. 63 - 74, [En línea] http://www.eumed.net/rev/rejie. COLAO MARín, P. A. (2015). "La enseñanza y aprendizaje del Derecho Tributario en Administración y Dirección de empresas: competencias, contenidos y referencia a la importancia del método del caso", REJIE: Revista Jurídica de Investigación e Innovación Educativa, núm. 12, pp. 65-80, http://www.eumed.net/rev/rejie. PÉREZ CÁZARES, M. E. (2012). "Teoría de los conceptos en la enseñanza del Derecho en base a casos concretos", Academia: Revista sobre enseñanza del Derecho, Buenos Aires, año X, núm. 20, pp. 31-49. DEL VALLE ARAmBURU, R. (2012). "Relaciones entre la enseñanza y el aprendizaje del derecho romano en la aplicación de los casos prácticos", Revista Electrónica del Instituto de Investigaciones Jurídicas y Sociales Ambrosio Lucas Gioja, año VI, núm. 8, pp. 7-26, http://www.derecho.uba.ar/revistagioja .

${ }^{51}$ Bueno Gallardo, E. (2013). "Formulación de una propuesta didáctica para la enseñanza-aprendizaje de la asignatura Derecho Financiero y Tributario I", Docencia y Derecho, Revista para la Docencia Jurídica Universitaria, núm. 7, pp. 17-18.
} 
desarrollar una tarea que luego se integra al resto. Utilizado en los Estados Unidos a partir de 1971, cada vez gana más adeptos.

Con esta relación no se agotan las ilimitadas metodologías pedagógicas con que puede contar el profesorado para incentivar a sus alumnos mayores y obtener mejores resultados. Solamente se ha querido mostrar algunos ejemplos para ilustrar variadas posibilidades que cada docente puede emplear.

\subsection{Utilización de los TICs.}

En Cuba existe bastante conciencia acerca de la validez en pedagogía internacionalmente reconocida- de las técnicas de la información y de la comunicación $(\mathrm{TICs})^{\mathbf{5 2}}$, de hecho se estimula su uso y es frecuente encontrar las aulas habilitadas con equipos que facilitan la impartición de la docencia con esas bondades.

El problema no se deslinda por ese camino sino por otros a él relacionados:

a) no todos los profesores están lo suficientemente diestros en la utilización de esos medios,

b) los alumnos no tienen suficiente accesibilidad a Internet, páginas webs, etc.,

c) existen dificultades con el suministro de energía eléctrica,

d) los estudiantes mayores se sienten limitados ante los problemas del transporte urbano lo cual afecta su asistencia a locales equipados con Internet, etc.

Todos estos asuntos apuntan en contra de su utilización, situación preocupante pues a muchos estudiantes les atraen la utilización de la web y el uso de las TICs, además, ello limita enriquecer sus conocimientos de forma atractiva, pues puede contribuir a estimular la lectura, actividad deficiente actualmente a pesar de ser tan importante. En Cuba esas dificultades han llevado a la creación de un Programa Nacional por la Lectura, considerado como un conjunto de acciones de carácter estratégico, proyectadas para un desarrollo a largo plazo y con participación y efectos tan amplios como la totalidad de la sociedad cubana ${ }^{53}$.

Cada vez más se imbrica la informática con la enseñanza del Derecho y se crean ambientes realmente virtuales para fomentar el conocimiento, que también han sido aprovechados para el aprendizaje semi presencial o no presencial $^{54}$, tan necesario ante las dificultades del transporte apuntadas anteriormente.

\footnotetext{
52 Restrepo Pimienta, J. L. y Forero Contreras, R. (2015). "Usos y prácticas de las técnicas de información y de la comunicación tic's en la enseñanza del derecho del trabajo", Revista Internacional y Comparada de Relaciones Laborales y Derecho del Empleo, ADAPT University Press, vol. 3, núm. 2, pp. $2-12$.

${ }^{53}$ LeÓn Pino, Y. y Ruiz Álvarez, M. M. (2016). "La promoción de lectura desde el trabajo comunitario de estudiantes de carreras pedagógicas del centro universitario municipal", Tlatemoani: Revista Académica de Investigación, núm. 21, http://www.eumed.net/rev/tlatemoani/21/tlatemoani21.pdf.

${ }^{54}$ Ridao MaRTín, J. (2016). "La enseñanza del Derecho Constitucional mediante las TIC. Un estudio de caso no-presencial", REJIE: Revista Jurídica de Investigación e Innovación Educativa, Núm.13, enero, pp. 49 -62, [En línea] http://www.eumed.net/rev/rejie
} 
Sería recomendable mejorar el equipamiento informático y crecer en su uso docente, como pudiera ser con el incremento en la elaboración de aplicaciones informáticas para teléfonos ${ }^{55}$, tableros de discusión, presencia de bases de datos legislativas al acceso de todos, la posibilidad de ejecutar exámenes virtuales ${ }^{56}$, la impartición de Diplomados para mejorar estos conocimientos ${ }^{57}$, pero también "adaptarse a nuevas formas de enseñanza, de introducir prácticas tuteladas por ordenador, de escribir en un blog para recibir los comentarios de los alumnos" Campus Virtual, de los foros de discusión, de tutorías virtuales, de test interactivos ${ }^{59}$, los cómics en la web ${ }^{60}$, etc.

\subsection{Tratamiento diferenciado de la enseñanza a mayores.}

A partir de este supuesto imprescindible, que constituye la apreciación más relevante del trabajo, debió iniciarse la exposición, pero como sobre esta base se cimentan todas las demás recomendaciones, se ha decidido colocarlo al final para cerrar la idea: la enseñanza de mayores tiene particularidades que deben ser tenidas en cuenta de forma prioritaria si se quieren lograr buenos resultados pedagógicos.

Para ello habrá de asumirse cambios de modos de actuar, inclusión de los métodos de enseñanza más adecuados, enfoques personalizados, en fin, ajustes que han traspasar las correcciones y acomodos usuales que no bastan, para potenciar el avance de las competencias y el fortalecimiento de las aptitudes de los educandos mayores.

Lo esencial será transformar conscientemente la manera tradicional del profesorado de brindar la información a los alumnos. La interacción entre ambos y la riqueza de los

\footnotetext{
${ }^{55}$ IÑIgo CorrozA, M. E. y SÁnCheZ- Ostiz GutiérRez, P. (2014). "Sobre el uso del método inductivo en la enseñanza del Derecho Penal”, REJIE: Revista Jurídica de Investigación e Innovación Educativa, núm.9, p. 14.

${ }^{56}$ VARGAS VASSEROT, C. (2009). "El método del caso en la enseñanza del Derecho: experiencia piloto de un piloto novel”, Refiedu: Revista de Formación e Innovación Educativa Universitaria, vol. 2, núm. 4, p. 197.

${ }^{57}$ Restrepo Pimienta, J. L. y Forero Contreras, R. (2015). "Usos y prácticas de las técnicas de información y de la comunicación tic's en la enseñanza del derecho del trabajo", Revista Internacional y Comparada de Relaciones Laborales y Derecho del Empleo, ADAPT University Press, vol. 3, núm. 2, p. 8.

58 SÁNChez DE Diego, M. (2010). "Una visión crítica a partir de la experiencia en la gestión modificación de planes de estudio en ciencias de la información”, en COTINO HUESO, Lorenzo y PRESNO LinerA, Miguel (coords.), Innovación educativa en Derecho constitucional. Reflexiones, métodos y experiencias de los docentes, PUV (Servicio de Publicaciones de la Universidad de Valencia), Valencia, p. 57, http://www.uv.es/derechos/innovacionconstitucional.pdf.

59 Presno, M. y Álvarez, L. (2010). "La creación de un entorno adecuado para el aprendizaje del Derecho constitucional", en Cotino Hueso, Lorenzo y PRESNO LINERA, Miguel (coords.), Innovación educativa en Derecho constitucional. Reflexiones, métodos y experiencias de los docentes, PUV (Servicio de Publicaciones de la Universidad de Valencia), Valencia, pp. 88-90, http://www.uv.es/derechos/innovacionconstitucional.pdf.

${ }^{60}$ Escalante Barreto, Caviedes Estanislao, Valoración pedagógica de la enseñanza del Derecho Penal General: una apuesta por el aprendizaje activo y colaborativo, Primera edición, Universidad Nacional de Colombia (Sede Bogotá), Facultad de Derecho, Ciencias Políticas y Sociales, Instituto Unidad de Investigaciones Jurídico - Sociales Gerardo Molina (UNIJUS), Bogotá, C. C., 2016, pp. 279 y ss.
} 
análisis permitirán el desarrollo de mejores habilidades con una superior formación intelectual, para garantizar un futuro desempeño profesional adecuado.

\section{Conclusiones.}

Es una tarea pueril el tratar de dictar reglas sobre cómo debe ocurrir el proceso de enseñanza aprendizaje para el alumnado mayor, aunque se reconozca la necesidad de un tratamiento diferenciado. Las estrategias cognitivas apuntadas deben considerarse solamente como ciertas pautas que pueden ser valoradas cuando así sean procedentes.

De más está reafirmar que ninguna de las recomendaciones tienen el objetivo de socavar construcciones mentales válidas o de convertirse en camisa de fuerza, todo lo contrario, solo constituyen una exposición de ideas para si así se considerase, puedan ser aplicadas de manera creativa, con conciencia de la diferencia, en función de las necesidades docentes.

Los tiempos cambian, es posible movilizar desde adentro, encontrar caminos pedagógicos alternativos para las situaciones que se presenten, construir dinámicamente sobre la base de los disímiles contextos a los que se hace frente, sin socavar una cosmovisión plena de valores como necesidad epistemológica.

En medio de nuestra compleja época, ningún criterio puede ser considerado excluyente. No tenemos opción para mantener una constante retroalimentación en un ambiente contextual pedagógico, por ello, será preciso investigar para asumir nuevos retos, incentivar distintas actividades docentes, contribuir a retener contenidos, hacer más dinámicas las clases, aceptar otras experiencias interesantes o exitosas, fomentar encuentros nacionales e internacionales sobre la temática, interrelacionar con diferentes disciplinas, propiciar estudios e investigaciones más sobre este sector del alumnado, etc., etc.

En fin, se propone una consideración holística de la enseñanza superior de mayores, con un criterio concientizado y diferenciado desde la concepción de los planes de estudio, la utilización de las Tics, los métodos de enseñanza, la preparación de los profesores, y cuantos más discernimientos propendan al fin último: lograr impartir una docencia acorde al momento actual.

\section{Bibliografía}

Alama Flores, C. M. (2015). "Hacia una didáctica de la metacognición”, Horizonte de la Ciencia, Junín, Perú, vol. 5, núm. 8, pp.77-86, [En línea] http://www.uncp.edu.pe/revistas/index.php/horizontedelaciencia/issue/view/9.

AMADIO, Massimo, et al. (2013). "Ejes de formación y enfoques curriculares", Oficina Internacional de Educación de la UNESCO (Ginebra, Suiza), [En línea] http://www.unesco.org/new/es/santiago/press-room/newsletters/newsletter-laboratoryfor-assessment-of-the-quality-of-education-llece/n12/training-areas-and-curriculumapproaches/ 
Bueno Gallardo, E. (2013). "Formulación de una propuesta didáctica para la enseñanza-aprendizaje de la asignatura Derecho Financiero y Tributario I", Docencia y Derecho, Revista para la Docencia Jurídica Universitaria, núm. 7, pp. 15-16.

Codina Felip, M. J. (2015). Neuroeducación en virtudes cordiales: cómo reconciliar lo que decimos con lo que hacemos, Barcelona, Ediciones Octaedro, S.L., pp. 91 y ss.

Colao Marín, P. A. (2015). "La enseñanza y aprendizaje del Derecho Tributario en Administración y Dirección de empresas: competencias, contenidos y referencia a la importancia del método del caso", REJIE: Revista Jurídica de Investigación $e$ Innovación Educativa, núm. 12, pp. 65-80, [En línea] http://www.eumed.net/rev/rejie.

Consolo, A. N. (2015). "El Arbitraje Comercial Internacional y los Moot Practice. Los métodos que se utilizan en la enseñanza de las Ciencias Jurídicas y Sociales", Derecho y ciencias sociales, La Plata, núm. 13, octubre, pp. 3-12.

Corredoira, L. (2010). "El uso de la IP TV en la docencia de Derecho de la Información", en Cotino Hueso, Lorenzo y PRESNO LinerA, Miguel (coords.), Innovación educativa en Derecho constitucional. Reflexiones, métodos y experiencias de los docentes, PUV (Servicio de Publicaciones de la Universidad de Valencia), Valencia, pp. 199 y ss., [En línea] http://www.uv.es/derechos/innovacionconstitucional

Cotino Hueso, L. (2010). "No casos, sino 'pasos' en la enseñanza de derechos fundamentales", en Cotino Hueso, Lorenzo y Presno Linera, Miguel (coords.), Innovación educativa en Derecho constitucional. Reflexiones, métodos y experiencias de los docentes, PUV (Servicio de Publicaciones de la Universidad de Valencia), Valencia, p. 102. [En línea] http://www.uv.es/derechos/innovacionconstitucional.pdf.

DAVison M, D. (2006). "La visión jeffersoniana de la educación jurídica", Academia. Revista sobre Enseñanza del Derecho, Buenos Aires, año IV, núm. 7, pp. 47-49.

Del Valle Aramburu, R. (2012). "Relaciones entre la enseñanza y el aprendizaje del derecho romano en la aplicación de los casos prácticos", Revista Electrónica del Instituto de Investigaciones Jurídicas y Sociales Ambrosio Lucas Gioja, año VI, núm. 8, pp. 7-26, [En línea] http://www.derecho.uba.ar/revistagioja

ElíAs MÉnDEZ, C. (2010). "Análisis de los textos y de la normativa de referencia en relación con el proceso de Bolonia", en Cotino Hueso, Lorenzo y PRESNo LinerA, Miguel (coords.), Innovación educativa en Derecho constitucional. Reflexiones, métodos y experiencias de los docentes, PUV (Servicio de Publicaciones de la Universidad de Valencia), Valencia, pp. 20-21, [En línea] http://www.uv.es/derechos/innovacionconstitucional

Escalante BARreto, Caviedes Estanislao, Valoración pedagógica de la enseñanza del Derecho Penal General: una apuesta por el aprendizaje activo y colaborativo, Primera edición, Universidad Nacional de Colombia (Sede Bogotá), Facultad de Derecho, Ciencias Políticas y Sociales, Instituto Unidad de Investigaciones Jurídico Sociales Gerardo Molina (UNIJUS), Bogotá, C. C., 2016, pp. 279 y ss. 
Escobar RocA, G. (2010). "Seis años de experiencia de formación virtual del programa regional de apoyo a las defensorías del pueblo de Iberoamérica", en Cotino Hueso, Lorenzo y Presno Linera, Miguel (coords.), Innovación educativa en Derecho constitucional. Reflexiones, métodos y experiencias de los docentes, PUV (Servicio de Publicaciones de la Universidad de Valencia), Valencia, pp. 180-184, [En línea] http://www.uv.es/derechos/innovacionconstitucional

GonZÁlez Rus, J. J. (2003). "Reflexiones sobre el futuro de la enseñanza del Derecho y sobre la enseñanza del Derecho en el futuro", Revista Electrónica de Ciencia Penal y Criminología, (en línea), núm. 05-r1, p. r1:1-r1:21, [En línea] http://criminet.ugr.es/recpc/recpc05-r1.pdf

Íñigo Corroza, M. E. y SÁnchez- Ostiz Gutiérrez, P. (2014). "Sobre el uso del método inductivo en la enseñanza del Derecho Penal", REJIE: Revista Jurídica de Investigación e Innovación Educativa, núm.9, p. 13.

KANDEL, V. N. (2010). "Cuando la universidad se enseña a sí misma. Notas sobre carrera docente academia", Revista Sobre Enseñanza del Derecho, Buenos Aires, Argentina, año VIII, núm. 16, pp. 231-239.

LARrauri Torroella, R. (2006). "La enseñanza del concepto de derecho desde la Filosofía del Derecho contemporánea”, Academia. Revista sobre Enseñanza del Derecho, Buenos Aires, año IV, núm. 7, pp. 159 y ss.

Lemes Batista, A. y CÁceres Giraud, A. (2006). "Enfoque de la interdisciplinaridad y transdisciplinaridad en la enseñanza de la Economía Política", Revista Iberoamericana de Educación, OEI, núm. 40/6, 15 de diciembre.

León Pino, Y. y Ruiz Álvarez, M. M. (2016). "La promoción de lectura desde el trabajo comunitario de estudiantes de carreras pedagógicas del centro universitario municipal”, Tlatemoani: Revista Académica de Investigación, núm. 21, abril, [En línea] http://www.eumed.net/rev/tlatemoani/21/tlatemoani21

MARTínez ALARCÓn, M. L. (2010). “Algunas reflexiones sobre la revisión del modelo docente universitario", en Cotino Hueso, Lorenzo y PRESNO Linera, Miguel (coords.), Innovación educativa en Derecho constitucional. Reflexiones, métodos y experiencias de los docentes, PUV (Servicio de Publicaciones de la Universidad de Valencia), Valencia, p. 49, [En línea] http://www.uv.es/derechos/innovacionconstitucional

Molina Saldarriaga, C. A. (2008). "Fundamentos teóricos y metodológicos del método clínico de enseñanza del derecho", Revista Facultad de Derecho y Ciencias Políticas, vol. 38, núm. 108, pp. 187-214.

NiETo, A. (2005). "La enseñanza académica del Derecho", Lex Nova, julio- septiembre, pp. 13, 27. 
Oliveros Aya, C. (2010). "El cine político: un recurso didáctico en la enseñanza del derecho constitucional", Diálogos de saberes: investigaciones y ciencias sociales, núm. 33 , pp. 245-260.

PAdrón Innamorato, M. y CÁceres Nieto, E. (2009). “Sociología jurídica y constructivismo: hacia una metodología para la determinación de la pensión alimenticia en el estado de Tabasco", Revista Latinoamericana de Derecho Social, núm. 9, pp. 122 y ss. [En línea] http://www.juridicas.unam.mx/publica/librev/rev/revlads/cont/9/art/art5

Palao Taboada, C. (2002). "La enseñanza del derecho en la Universidad: presente y futuro", AFDUAM, núm. 6, pp. 127-139.

Peñas Moyano, M. J. et al. (2010). "Métodos cooperativos en la enseñanza del derecho: una aproximación al derecho mercantil en las aulas de facultades no jurídicas", Revista de educación y derecho. EDUCATION AND LAW REVIEW, núm. 2, abril septiembre, pp. 1-21.

Pérez CÁzAres, M. E. (2012). "Teoría de los conceptos en la enseñanza del Derecho en base a casos concretos", Academia: Revista sobre enseñanza del Derecho, Buenos Aires, año X, núm. 20, pp. 31-49.

Pires Pinto Sica, L. P. et al. (2012). "Reflexiones sobre enseñanza del Derecho y evaluación en el contexto brasileño", Academia. Revista sobre enseñanza del Derecho, Buenos Aires, año X, núm. 20, pp. 64-65.

Presno, M. y Álvarez, L. (2010). "La creación de un entorno adecuado para el aprendizaje del Derecho constitucional", en Cotino Hueso, Lorenzo y PRESNO LINERA, Miguel (coords.), Innovación educativa en Derecho constitucional. Reflexiones, métodos y experiencias de los docentes, PUV (Servicio de Publicaciones de la Universidad de Valencia), Valencia, pp. 88-90, [En línea] http://www.uv.es/derechos/innovacionconstitucional

Restrepo Pimienta, J. L. y Forero Contreras, R. (2015). “Usos y prácticas de las técnicas de información y de la comunicación tic's en la enseñanza del derecho del trabajo", Revista Internacional y Comparada de Relaciones Laborales y Derecho del Empleo, ADAPT University Press, vol. 3, núm. 2, pp. 2-12.

Ridao Martín, J. (2016). "La enseñanza del Derecho Constitucional mediante las TIC. Un estudio de caso no-presencial”, REJIE: Revista Jurídica de Investigación e Innovación Educativa, Núm.13, enero, pp. 49 - 62, [En línea] http://www.eumed.net/rev/rejie

Rocha NúÑEZ, E. (2011). "Aplicación del método ABP y la clínica procesal en la enseñanza aprendizaje del Derecho Procesal Mercantil”, RIDE: Revista Iberoamericana para la Investigación y el Desarrollo Educativo, vol. 1, núm. 2, enero - junio, p. 163. 
RUBio NúÑEZ, R. (2010). "La enseñanza virtual de un Derecho global”, en Cotino Hueso, Lorenzo y PRESNO LinerA, Miguel (coords.), Innovación educativa en Derecho constitucional. Reflexiones, métodos y experiencias de los docentes, PUV (Servicio de Publicaciones de la Universidad de Valencia), Valencia, p. 34, [En línea] http://www.uv.es/derechos/innovacionconstitucional

SAlazAR BeníteZ, O. (2010). "Estrategias y herramientas para la enseñanza del derecho constitucional", Docencia y Derecho, núm. 1, p. 9.

SÁNCHEZ DE DiEGo, M. (2010). "Una visión crítica a partir de la experiencia en la gestión modificación de planes de estudio en ciencias de la información", en Cotino Hueso, Lorenzo y PRESNo LINERA, Miguel (coords.), Innovación educativa en Derecho constitucional. Reflexiones, métodos y experiencias de los docentes, PUV (Servicio de Publicaciones de la Universidad de Valencia), Valencia, p. 57, [En línea] http://www.uv.es/derechos/innovacionconstitucional

SÁnCHEZ RuIZ, M. (2013). "Cuánto y cómo enseñar derecho mercantil en el grado en relaciones laborales y recursos humanos", Trabajo. Revista andaluza de relaciones laborales, Universidad de Huelva, Huelva, núm. 29, pp. 85-99.

Serrano Pérez, M. M. y Martí SÁnchez, J. M. (2010). "La planificación y desarrollo compartido de competencias", en Cotino Hueso, Lorenzo y PRESNO LINERA, Miguel (coords.), Innovación educativa en Derecho constitucional. Reflexiones, métodos y experiencias de los docentes, PUV (Servicio de Publicaciones de la Universidad de Valencia), Valencia, p. 66, [En línea] http://www.uv.es/derechos/innovacionconstitucional

TAJADURA, J. (2010). "La utilización de medios audiovisuales en las clases prácticas de Derecho Constitucional”, en Cotino Hueso, Lorenzo y PRESNo LinerA, Miguel (coords.), Innovación educativa en Derecho constitucional. Reflexiones, métodos y experiencias de los docentes, PUV (Servicio de Publicaciones de la Universidad de Valencia), Valencia, [En línea] http://www.uv.es/derechos/innovacionconstitucional , pp. 192-198.

Torres Villarreal, M. L. (2013). "La enseñanza clínica del derecho: una forma de educación para el cambio social. La experiencia del grupo de acciones públicas de la Universidad del Rosario", Revista Facultad de Derecho y Ciencias Políticas, Medellín, vol. 43, núm. 119, enero-junio, pp. 725-726.

VARgas VAsserot, C. (2009). "El método del caso en la enseñanza del Derecho: experiencia piloto de un piloto novel", Refiedu: Revista de Formación e Innovación Educativa Universitaria, vol. 2, núm. 4, p. 205.

VILA Ramos, B. (2011). "Una propuesta práctica de innovación docente: La enseñanza-aprendizaje-evaluación del Derecho Constitucional”, Indret: Revista para el Análisis del Derecho, núm. 1, p. 28. 
Weinstein, J. (1998). "La búsqueda del tesoro perdido. Educación y Juventud en América Latina”, Boletín Proyecto Principal de Educación en América Latina y el Caribe, núm. 45, abril, pp. 71-87.

Zumaquero Gil, L. (2016). "Cómo enseñar derecho civil en la titulación de grado en Turismo a través del estudio de casos", REJIE: Revista Jurídica de Investigación e Innovación Educativa, núm. 13, pp. 63 - 74, [En línea] http://www.eumed.net/rev/rejie 